\title{
Prediction of Human Nonlinear Pharmacokinetics of a New Bcl-2 Inhibitor Using PBPK Modeling and Interspecies Extrapolation Strategy ${ }^{\llbracket}$
}

\author{
Philippe B. Pierrillas, ${ }^{1}$ Emilie Henin, ${ }^{1}$ Kathryn Ball, ${ }^{2}$ Julien Ogier, ${ }^{3}$ Magali Amiel, \\ Laurence Kraus- Berthier, Marylore Chenel, François Bouzom, ${ }^{4}$ and Michel Tod
}

Equipe mixte de recherche 3738, Ciblage Thérapeutique en Oncologie, Faculté de Médecine et de Maïeutique Lyon-Sud Charles Mérieux, Université Claude Bernard Lyon 1, Oullins, France (P.P., E.H., M.T.); Pharmacie Hôpital de la Croix Rousse, Hospices Civils de Lyon, Lyon, France (M.T.); Centre de Pharmacocinétique et Métabolisme, Technologie Servier, Orléans, France (P.P., F.B.);

Clinical Pharmacokinetics and Pharmacometrics Division, Servier, Suresnes, France (K.B., J.O., M.A., M.C.); and Institut de Recherches Internationales Servier, Oncology R\&D Unit, Suresnes, France (L.K-B.)

Received November 27, 2018; accepted March 26, 2019

\section{ABSTRACT}

S 55746 ((S)-N-(4-hydroxyphenyl)-3-(6-(3-(morpholinomethyl)-1,2,3,4tetrahydroisoquinoline-2-carbonyl)benzo[d][1,3]dioxol-5-yl)-N-phenyl5,6,7,8-tetrahydroindolizine-1-carboxamide) is a new selective $\mathrm{Bcl}-2$ (B-cell lymphoma 2) inhibitor developed by Servier Laboratories and used to restore apoptosis functions in cancer patients. The aim of this work was to develop a translational approach using physiologically based (PB) pharmacokinetic (PK) modeling for interspecies extrapolation to anticipate the nonlinear PK behavior of this new compound in patients. A PBPK mouse model was first built using a hybrid approach, defining scaling factors (determined from in vitro data) to correct in vitro clearance parameters and predicted $K p$ (partition coefficient) values. The qualification of the hybrid model using these empirically determined scaling factors was satisfactorily completed with rat and dog data, allowing extrapolation of the PBPK model to humans. Human PBPK simulations were then compared with clinical trial data from a phase 1 trial in which the drug was given orally and daily to cancer patients. Human PBPK predictions were within the $95 \%$ prediction interval for the eight dose levels, taking into account both the nonlinear dose and time dependencies occurring in S 55746 kinetics. Thus, the proposed PK interspecies extrapolation strategy, based on preclinical and in vitro information and physiologic assumptions, could be a useful tool for predicting human plasma concentrations at the early stage of drug development.

\section{Introduction}

The development of a new chemical entity is a long and expensive process. According to several studies (Kola and Landis, 2004), the rate of approval in the field of oncology after entry into phase 1 is approximately 5\%, among the lowest of all therapeutic areas. At the

E.H. was funded by Fondation Synergie Lyon Cancer and La Ligue Nationale contre le Cancer. This work is part of a Ph.D. project (P.P.), granted by Servier Laboratories.

${ }^{1}$ Current affiliation: Calvagone, Liergues, France.

${ }^{2}$ Current affiliation: Clinical Pharmacology, Drug Metabolism and Pharmacokinetics, Medlmmune, Cambridge, United Kingdom.

${ }^{3}$ Current affiliation: Translational DMPK \& Safety, IPSEN Innovation, Les Ulis, France.

${ }^{4}$ Current affiliation: UCB Biopharma, Development Sciences, Braine l'Alleud, Belgium.

https://doi.org/10.1124/dmd.118.085605.

S This article has supplemental material available at dmd.aspetjournals.org. beginning of the 1990s, the main reasons for the high attrition rate were a lack of efficacy and unfavorable pharmacokinetics (PKs). For this reason, the improvement of drug selection at the earliest stage of development based on PK properties (absorption, distribution, metabolism, and excretion) has been encouraged, and the extrapolation of preclinical data to humans has been performed much earlier in the development process.

In this way, preclinical information can be a precious resource for predicting the behavior of a compound in humans and therefore can be decisive for "go/no go" decisions. Several methods with different degrees of complexity have been described to predict human PKs from nonclinical settings. The most empirical approach for interspecies PK extrapolation is based on the prediction of human PK parameters (clearance, volume of distribution) by allometric scaling (Boxenbaum, 1982; Mordenti, 1986; West et al., 2002; Tang and Mayersohn, 2006) and has been widely used in recent decades. Other more sophisticated strategies have also been developed (Dedrick, 1973; Wajima et al., 2004), based on the assumption that

ABBREVIATIONS: AFE, average fold error; AUC, area under the curve; $\mathrm{Bcl}-2$, B-cell lymphoma 2; $\mathrm{CL}_{\text {int }}$, intrinsic clearance; $\mathrm{f}_{\mathrm{u}, \mathrm{p}}$, unbound fraction in plasma; HLM, human liver microsome; $\mathrm{K}_{a p p}$, concentration that produces the half-maximal rate of inactivation; $\mathrm{K}_{\text {inact }}$, maximum inactivation rate constant; $\mathrm{K}_{m}$, drug concentration in plasma corresponding to $50 \%$ of the maximum rate of elimination; $\mathrm{Kp}$, partition coefficient; LC-MS/MS, liquid chromatography coupled with mass spectrometry in tandem; Papp, apparent permeability; PB, physiologically based; PBPK, physiologically based pharmacokinetics; PK, pharmacokinetic; $R_{b l: p}$, blood-to-plasma concentration ratio; RMSE, root mean square error; $\mathrm{S} 55746$, (S)-N-(4hydroxyphenyl)-3-(6-(3-(morpholinomethyl)-1,2,3,4-tetrahydroisoquinoline-2-carbonyl)benzo[d][1,3]dioxol-5-yl)- $N$-phenyl-5,6,7,8-tetrahydroindolizine1-carboxamide; SCID, severe combined immunodeficient; SF, scaling factor; $\mathrm{V}_{m}$, in vivo maximum rate of elimination. 
concentration-time profiles of a drug from a variety of species could be superimposed when the curve axes were normalized (by mean residence time, body weight, or dose per kilogram). However, those techniques can be applied only to drugs with linear kinetics and thus have a limited range of applications.

With the progress of in vitro experiments and the availability of microsomes and hepatocytes from different species, human clearance parameters could be better determined thanks to the development of in vitro/in vivo extrapolation (Houston and Carlile, 1997; Houston, 2013). The use of PBPK (physiologically based PK) modeling (Rostami-Hodjegan et al., 2012) can be an attractive strategy for predicting drug PK thanks to the differentiation of system-specific parameters from drug-specific parameters. Unlike empirical and classic compartmental models, which can be useful to describe data, PBPK models use physiologic concepts that facilitate interspecies extrapolation. In a PBPK model, compartments represent tissues or organs; their volumes are set to the real physical volumes, and the different organ-specific blood flows are also considered. In this approach, the disposition profiles are predicted from the physicochemical properties of the compound and the species-specific physiologic parameters (Espié et al., 2009; Jones and Rowland-Yeo, 2013).

Most interspecies extrapolation studies deal with compounds presenting linear PK processes, and few studies have tried to scale the kinetics of drugs exhibiting nonlinear behavior (Dong et al., 2011; Chen et al., 2013) from preclinical studies to clinical trials. In those studies, a Michaelis-Menten equation (Michaelis et al., 2011) has been used to describe the nonlinear phenomenon, and correlations have been calculated using a power law model (Dedrick, 1973) (according to body weight) for the $V_{m}$ (maximum rate of elimination) and $K_{m}$ (drug concentration corresponding to $50 \%$ of the maximum rate of elimination) parameters to predict human parameters. Even if acceptable predictions have been made at some doses, though, difficulties have still been highlighted regarding the ability to capture both linear and nonlinear processes simultaneously (Dong et al., 2011; Chen et al., 2013).

This study focused on the compound S 55746 [(S)- $N$-(4-hydroxyphenyl)3-(6-(3-(morpholinomethyl)-1,2,3,4-tetrahydroisoquinoline-2-carbonyl)benzo[d][1,3]dioxol-5-yl)- $N$-phenyl-5,6,7,8-tetrahydroindolizine-1carboxamide], which was identified as a new Bcl-2 (B-cell lymphoma 2) inhibitor. Bcl-2 family proteins largely mediate the mitochondrial apoptotic pathway (Bagci et al., 2006), and, in many cancers, apoptosis is affected by Bcl-2 antiapoptotic protein overexpression (Cosulich et al., 1997; Ottilie et al., 1997), impairing the ability of the cell to undergo normal apoptosis (Reed, 2004). Therefore, by inhibiting the Bcl-2 protein, S 55746 can reactivate apoptosis and decrease tumor growth. S 55746 has shown nonlinear PKs during preclinical studies, which presents challenges for interspecies scaling of its PKs. The aim of this work therefore was 1) to propose a PK extrapolation strategy based on PBPK modeling, 2) to predict its PK behavior in humans based on preclinical studies, and 3) to compare our predictions to the data from the first-in-human trial of S 55746 .

\section{Materials and Methods}

\section{In Vitro Methods}

Protein Binding. S 55746 (Fig. 1) was provided by Servier Laboratories. The unbound fraction in plasma $\left(\mathrm{f}_{\mathrm{u}, \mathrm{p}}\right)$ of $[14 \mathrm{C}]-\mathrm{S} 55746$ was determined in animals and humans by dialysis using the Rapid Equilibrium Dialysis Device System (Thermo Fisher Scientific, Waltham, MA) at concentrations of 500, 5000, and 50,000 ng/ml for all species. Aliquots of spiked plasma were placed into the sample chamber,

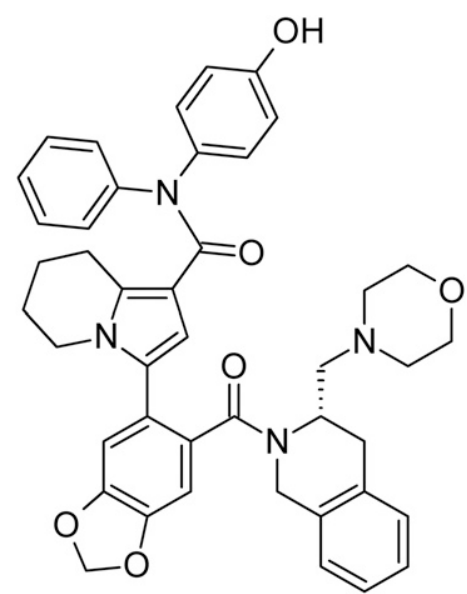

Fig. 1. Chemical structure of S 55746 .

and samples of nonspiked dialysis buffer were added to the buffer chamber. The plates were covered and incubated at $37^{\circ} \mathrm{C}$ at $500 \mathrm{rpm}$ on an orbital shaker (for $1,3,4$, and 5 hours). All incubations were performed in triplicate and analyzed by liquid scintillation counting.

Blood-to-Plasma Partition. The blood-to-plasma concentration ratio $\left(R_{b l: p}\right)$ was determined in triplicate per concentration for all species. Prior to spiking blood, a small aliquot of blood was taken for the determination of the hematocrit. Spiked blood samples were incubated on a roller mixer for 1 hour at $37^{\circ} \mathrm{C}$ and then centrifuged at room temperature. Finally, plasma was transferred into appropriate tubes to be analyzed by liquid scintillation counting to allow the determination of drug concentration. The $R_{b l: p}$ was calculated using the following equation:

$$
R_{b l: p}=\frac{C_{b l}}{C_{p}}
$$

where $C_{b l}$ and $C_{p}$ are, respectively, the drug concentration in blood and plasma.

Microsome and Hepatocyte Clearance Determination. Microsomal incubations were carried out for different times $\left(0,7,17,30\right.$, and 60 minutes) at $37^{\circ} \mathrm{C}$ with human liver microsomes (HLMs) with S 55746 at a final concentration of 0.1 and $40 \mu \mathrm{M}$. Reactions were initiated by the addition of NADPH after a 10 -minute preincubation time. During incubation, aliquots were sampled at each incubation time and the enzymatic reaction was stopped by protein precipitation using methanol.

For hepatocytes, S 55746 was incubated for different time points $(0,5,10,20$, 30, 60, and 100 minutes) in William's EC Medium (Thermo Fisher Scientific). Reactions were initiated by the addition of S 55746. During incubation, aliquots were sampled at each incubation time, and the enzymatic reaction was stopped by protein precipitation using acetonitrile.

Samples were then centrifuged and the supernatants transferred to analysis plates, followed by analysis using liquid chromatography coupled with mass spectrometry in tandem (LC-MS/MS).

The $K_{m}$ and $V_{m}$ parameters of S 55746 in HLMs and in hepatocytes were determined via substrate disappearance kinetics. Since the enzyme activity decreases with time, $V_{m}$ can be described by:

$$
V_{m}=V_{m 0} \cdot e^{-k_{d} \cdot t}
$$

where $V_{m 0}$ is the initial $V_{m}$ and $k_{d}$ is the inactivation constant for the degradation of enzyme activity.

The model used to describe the disappearance of the substrate $(S)$ was therefore:

$$
\frac{d S}{d t}=-\frac{V_{m} \cdot[S]}{K_{m}+[S]} .
$$

The data were analyzed with the nonlinear analysis software WinNonlin version 5.1 (Pharsight Corporation). The $K_{m}$ and $V_{m}$ parameters were estimated with the inclusion of microsomal binding data. 
The determination of the intrinsic clearance was calculated using the following formulas according to microsome or hepatocyte experiments:

$$
\begin{gathered}
C L_{\text {int,vitro,mic } / \text { hep }}=\frac{V_{m}}{K_{m}+C_{u}} \\
C L_{\text {int, vivo }}=C L_{\text {int,vitro,mic }} \cdot M P P G L \cdot \text { Liver Weight }
\end{gathered}
$$

where $C_{u}$ is the unbound concentration, $C L_{\text {int,vivo }}$ is the intrinsic clearance for the whole organ, $C L_{\text {int,vitro,mic }}$ is the intrinsic clearance determined with the microsomal system, and MPPGL represents the microsomal protein per gram of liver of the relevant species, and:

$$
C L_{\text {int }, \text { vivo }}=C L_{\text {int,vitro,hep }} \cdot H P G L \cdot \text { Liver Weight }
$$

where $C L_{\text {int, vitro, hep }}$ is the intrinsic clearance measured by the hepatocyte system and HPGL represents the number of hepatocytes per gram of liver.

Identification of Enzymes Involved in Human Hepatic Metabolism. Cytochrome contribution was determined via clearance experiments with $\mathrm{S}$ 55746 and recombinant human cytochromes CYP1A2, CYP2C8, CYP2C9, CYP2C19, CYP2D6, and CYP3A4. S 55746 was incubated with each individual recombinant human cytochrome for $0,7,17,30$, or 60 minutes.

The relative contribution of each cytochrome enzyme to the oxidative part of the microsomal clearance of S 55746 was estimated by comparing the $C L_{i n t}$ of each individual recombinant human cytochrome $\left(C L_{i n t}, r h C Y P\right)$ to the sum of individual recombinant human cytochrome $C L_{\text {int }}\left(\sum \mathrm{CL}_{\mathrm{int}}, r h C Y P\right)$ :

$$
f m(\%)=\frac{C L_{i n t}, r h C Y P}{\sum C L_{i n t}, r h C Y P} .
$$

A relative activity factor (RAF) was estimated from the ratio of the $C L_{i n t}$ values for cytochrome-specific probe substrates using recombinant human cytochrome and using HLMs using the following equation:

$$
R A F=\frac{C L_{i n t}, r h C Y P}{C L_{i n t}, H L M}
$$

The concentration of bactosomes to be used in the incubation was calculated from the concentration of HLMs used as the standard for intrinsic clearance measurement, using the following equation:

$$
[r h C Y P]=\frac{[H L M]}{R A F}
$$

where $[r h C Y P]$ is the bactosomal protein concentration and $[H L M]$ is the microsomal protein concentration.

Supernatants were analyzed using a TurboFlow LC-MS/MS Platinum Mass Spectrometer (Thermo Fisher Scientific) to assay S 55746 concentrations. Orthogonal assays using microsomes and CYP inhibitors were conducted to confirm the results.

Assessment of Mechanism-Based Inhibition in HLMs. The possible existence of mechanism-based inhibition (Obach et al., 2007) of CYP3A4 was also investigated. HLMs were prewarmed with $\mathrm{S} 55746(0,0.25,0.5,1,2,3,10$, and $30 \mu \mathrm{M}$ ) for 5 minutes at $37^{\circ} \mathrm{C}$. The preincubation was initiated by the addition of NADPH or buffer, and at different preincubation times $(0,2,4,6,8,12$, and 14 minutes) an aliquot of the preincubate was collected and added to a vial containing midazolam (Sekiguchi et al., 2011). After an incubation of 7 minutes, reactions were terminated by the addition of methanol. Samples were placed on ice before centrifugation. Supernatants were analyzed using LC-MS/MS for the measurement of hydroxymidazolam concentration. Calculations and regression analysis were performed using Excel 2003 (Microsoft Corporation, Redmond, WA) and Xlfit4 (IDBS UK, Guildford, UK).

A value for $k_{\text {inact }}$ (maximum inactivation rate constant) and $K_{a p p}$ (concentration that produces the half-maximal rate of inactivation) was determined by nonlinear regression analysis. The data were fitted to the following equation:

$$
k_{\text {obs }}=\frac{k_{\text {inact }} \cdot[I]}{K_{\text {app }}+[I]}
$$

where $k_{o b s}$ is the slope observed when representing the natural logarithm of the percentage of remaining activity against the preincubation time and $[I]$ is the inhibitor concentration.
Induction. Isolated human hepatocytes cultured on type I collagen plates were used to assess the induction potential of S 55746. For 3 days, these human hepatocytes were treated with $\mathrm{S} 55746$ at $0.1,1,2.5$, 5 , or $10 \mu \mathrm{M}$ omeprazole (a known inducer of human CYP1A), phenobarbital (a known inducer of human CYP2B6), or rifampicin (a known inducer of human CYP3A). After 3 days, the hepatocytes were incubated with the cytochrome $\mathrm{P} 450$ substrates phenacetin (CYP1A2), bupropion (CYP2B6), and midazolam (CYP3A4) for the assessment of enzyme activity.

After the incubation, hepatocytes were harvested to isolate RNA, analyzed by quantitative reverse-transcription polymerase chain reaction. The obtained activities were compared with those of control cultures not exposed to the test compounds or to the reference cytochrome inducers. The results were expressed as fold induction compared with the control conditions or as a percentage of the reference inducer cytochrome activity or mRNA expression increase. When a concentration-dependent increase of cytochrome mRNA was observed and a maximum fold change was reached during the experiments, the data (fold induction) were processed for the determination of induction parameters (maximal induction, maximal fold induction, and the concentration at which there is a $50 \%$ maximal induction effect) by nonlinear regression fitting of the data.

Apparent Permeability Determination. The Caco-2 cell line (Hilgers et al., 1990) was cultured in Dulbecco's modified Eagle's medium containing $10 \%$ (v/v) of fetal calf serum, 1\% L-glutamine, nonessential amino acids, antibiotics, and amphotericin. Cells were seeded at 90,000 cells $/ \mathrm{cm}^{2}$ onto a microporous membrane of 24-multiwell insert plates so that apical (A) and basolateral (B) media would be separated by the cell monolayer. Cells were maintained at $37^{\circ} \mathrm{C}$ in an atmosphere of $95 \%$ air and $5 \% \mathrm{CO}_{2}$ and used between 21 and 30 days postseeding. S 55746 was incubated at $10 \mu \mathrm{M}$ in the apical chamber. Samples (duplicate) were collected at 30 and 90 minutes in the basolateral chamber and analyzed by LC-MS/MS. Reference compounds for apparent permeability $\mathrm{P}_{\text {арр А-в }}$ (cimetidine, propranolol) were used to determine the calibration curve of the Caco- 2 model.

Table 1 shows the in vitro and in silico parameters for all species.

\section{PBPK Modeling}

The PBPK modeling strategy was based on the use of either in vitro or in vivo data (according to a retrograde approach) depending on the quality of the predictions (Peters, 2008). Simcyp software, versions 13 and 14 (Jamei et al., 2009a, 2013) was used to perform PBPK modeling.

Distribution. In the full PBPK distribution model, we assumed that the drug distributes instantaneously and homogeneously within each tissue and that its uptake in each tissue is limited by the blood flow (perfusion limited). The rate of change of the drug in a noneliminating tissue is described by the following differential equation (Nestorov, 2003; Jones et al., 2009):

$$
V_{T} \cdot \frac{d C_{T}}{d t}=Q_{T} \cdot\left(C_{a, b l}-C_{v, b l, T}\right)=Q_{T} \cdot\left(C_{a, b l}-\frac{C_{T}}{K p_{T: b l}}\right)
$$

where $Q_{T}$ is the tissue blood flow and $V_{T}$ is the volume of the tissue. The subscript $T$ denotes the tissue, the subscript $a$ represents the artery, $b l$ denotes the blood, and $v$ denotes the vein. $C_{a, b l}$ and $C_{v, b l, T}$ refer to the concentration in the arterial blood entering the tissue and the concentration in the venous blood leaving the tissue, respectively, and $K p_{T: b l}$ is the partition coefficient between the tissue and the blood.

It has been considered that the drug might be eliminated only from liver where the rate of change of the drug can be expressed by:

$$
V_{h} \cdot \frac{d C_{h}}{d t}=Q_{h} \cdot\left(C_{a, b l}-C_{v, b l, h}\right)-C L_{i n t, v i v o} \cdot C_{u, h}
$$

where the subscript $h$ denotes the liver, $C L_{\text {int,vivo }}$ denotes the intrinsic in vivo clearance, and $C_{u, h}$ denotes the $C_{u}$ in the liver.

The equations of Poulin and Theil (2002a,b), modified by Berezhkovskiy (2004), and the equations of Rodgers et al. (2005a,b) and Rodgers and Rowland $(2006,2007)$ were compared with predicted $K p_{T: p}$ values from physicochemical parameters and the resulting volume of distribution in plasma:

$$
V_{S S}=V_{b l} \cdot R_{b l: p}+\sum V_{T} \cdot K p_{T: p}
$$

Clearance. The well-stirred model (Rowland et al., 1973; Pang and Rowland, 1977) was used to determine hepatic clearance $C L_{h, b l}$ from the intrinsic clearance $C L_{i n t}$, the hepatic blood flow $\mathrm{Q}_{\mathrm{h}}$, and the unbound fraction in blood $f_{u, b l}$ : 
TABLE 1

Characteristics of the S 55746 compound

\begin{tabular}{|c|c|c|c|c|c|}
\hline Parameters & & Mouse & Rat & Dog & Human \\
\hline \multicolumn{6}{|l|}{ Drug-dependent parameters } \\
\hline $\mathrm{pKa}$ & Acidic: 9.7/Base: 6.9 & & & & \\
\hline $\log \mathrm{P}$ & 4.4 & & & & \\
\hline Molecular weight $(\mathrm{g} / \mathrm{mol})$ & 710.8 & & & & \\
\hline \multicolumn{6}{|l|}{ Species-dependent parameters $(\%)$} \\
\hline $\mathrm{f}_{\mathrm{u}, \mathrm{p}}$ & & 1.50 & 2.77 & 1.78 & 2.50 \\
\hline \multicolumn{6}{|l|}{ In vitro metabolic parameters } \\
\hline \multicolumn{6}{|l|}{ Microsome parameters } \\
\hline$V_{m}(\mathrm{pmol} / \mathrm{min}$ per milligram protein $)$ & & 1310 & 4260 & $\mathrm{n} / \mathrm{a}$ & 1070 \\
\hline$K_{m}(\mu \mathrm{M})$ & & 0.44 & 1.54 & $\mathrm{n} / \mathrm{a}$ & 0.625 \\
\hline \multicolumn{6}{|l|}{ Hepatocyte parameters } \\
\hline$V_{m}\left(\mathrm{pmol} / \mathrm{min}\right.$ per $10^{6}$ hepatocytes $)$ & & 627 & $\mathrm{n} / \mathrm{a}$ & 132 & 283.8 \\
\hline$K_{m}(\mu \mathrm{M})$ & & 3.4 & $\mathrm{n} / \mathrm{a}$ & 0.8 & 1.08 \\
\hline \multicolumn{6}{|l|}{ Metabolic pathway in humans } \\
\hline Clearance due to cytochromes & $85 \%$ & & & & \\
\hline Contribution of cytochromes & $97 \%$ due to CYP3A4 & & & & \\
\hline \multicolumn{6}{|l|}{ MBI parameters } \\
\hline$k_{\text {inact }}(1 / \mathrm{h})$ & 12.6 & & & & \\
\hline$K_{a p p}(\mu \mathrm{M})$ & 1.55 & & & & \\
\hline \multicolumn{6}{|l|}{ Induction parameters } \\
\hline Ind max & 10.41 & & & & \\
\hline Ind $\mathrm{C} 50(\mu \mathrm{M})$ & 3.03 & & & & \\
\hline
\end{tabular}

Ind C50, concentration at which there is a 50\% maximal induction effect; Ind max, maximum induction; MBI, mechanism-based inhibition.

$$
C L_{h, b l}=\frac{Q_{h} \cdot f_{u, b l} \cdot C L_{i n t, v i v o}}{Q_{h}+f_{u, b l} \cdot C L_{\text {int }, v i v o}}
$$

where $f_{u, b l}$ was calculated by dividing the $\mathrm{f}_{\mathrm{u}, \mathrm{p}}$ by the blood-to-plasma ratio.

Oral Absorption. The oral absorption in humans was predicted using the ADAM (advanced dissolution, absorption, and metabolism) model (Jamei et al., 2009b; Patel et al., 2014) implemented in Simcyp. The ADAM model is a multicompartmental gastrointestinal transit model. The gastrointestinal tract is treated as a one-stomach, seven-small-intestine, and one-colon compartment, and the drug can exist in several states simultaneously: unreleased, undissolved, dissolved, or degraded. In this model, data from the $\mathrm{P}_{\mathrm{app}}$ experiment are used to predict the effective permeability across the enterocyte (Sun et al., 2002), and the integration of the dissolution profile of the formulation can be used to determine the fraction of the pharmaceutically active compound released from the formulation over time.

Strategy for Interspecies Qualification. Before performing PBPK simulations in humans, a qualification exercise was performed on different preclinical species. For this qualification with rat and dog PBPK models, the mouse physiologic model parameters were replaced with the corresponding values for rat and dog, and the drug-specific parameters measured in mouse-specific in vitro systems (e.g., $C L_{i n t}, \mathrm{f}_{\mathrm{u}, \mathrm{p}}$ ) were replaced with the corresponding values measured in rat and dog in vitro systems. Simulations were carried out based on the dose and administration schedule in the in vivo studies, and the rat and dog PBPK model predictions were evaluated for their goodness of prediction based on the model evaluation criteria described below (see Prediction Performances and Parameter Analyses). Intravenous PK data from two preclinical species, rat and dog, were available to qualify the PBPK approach and to validate interspecies extrapolation.

\section{In Vivo Preclinical PK Studies}

All of the animal experiments were reviewed and approved by the ethical committee, and were in general accordance with the animal health and welfare guidelines; it was also ensured there were no other alternative methods than live animals to achieve the study objectives. Severe combined immunodeficient (SCID) female mice, male Wistar rats, and male beagle dogs from Charles River Laboratories were used for PK studies. The oral formulations were different in each species, so only intravenous studies were considered in preclinical species (mouse, rat, dog) to better characterize clearance and distribution processes.

Mouse Studies. SCID mice (female) were treated with S 55746 by intravenous bolus of $1,3,10$, or $25 \mathrm{mg} / \mathrm{kg}$. Blood samples (from the tail or saphenous vein) between 0.083 and 30 hours after administering a dose were collected in two to five mice per time point.
Rat Studies. PK studies in Wistar rat (male) were conducted after an intravenous infusion administered at the following doses: $0.5,1.5$, or $5 \mathrm{mg} / \mathrm{kg}$. Blood samples (from the tail vein) were collected from 0.17 to 24 hours after administration. Three animals were used for each dose level.

Dog Studies. In PK studies in Beagle dog (male), S 55746 was given after intravenous administration of a single bolus. Doses of 0.2 and $1.5 \mathrm{mg} / \mathrm{kg}$ were administered to dogs. S 55746 was quantified from 0.17 to 24 hours after administration. Data from three dogs were available for each dose.

\section{Clinical Study}

Clinical protocols were approved by the French regulatory agency (Agence Nationale de Sécurité du Médicament), and all subjects gave written informed consent for participation in the clinical trials. Dose escalation in the phase I clinical trial was driven by the continual reassessment method (GarrettMayer, 2006).

S 55746 was given orally once a day to fasted cancer patients (non-Hodgkin lymphoma, chronic lymphocytic leukemia, and acute myeloid leukemia) for a period of 21 days at different doses $(100,200,300,400,500,700,900$, and $1100 \mathrm{mg}$ ). Blood samples were collected over 24 hours on the first and eighth days of administration.

\section{Bioanalytical Method}

The drug concentrations in plasma were determined using LC-MS/MS. For this bioanalytical method, samples were thawed at room temperature, vortex mixed, and centrifuged. Aliquots of samples were added to the Ostro (Waters, Milford, MA) sample preparation plate with the internal standard ( $N-(4-\{2-[(3 \mathrm{aS}, 9 \mathrm{bR})-$ 8-cyano-1,2,3,3a,4,9b-hexahydrochromeno [3,4-c]pyrrol-2-yl]ethyl\}phenyl)acetamide, hydrochloride) to remove proteins and phospholipids. Plates were mixed at room temperature. After elution in 96-well plate, $100 \mu \mathrm{l}$ of the eluate was mixed and injected directly into the LC-MS/MS system. Analytes were separated using a pentafluorophenyl column $(100 \times 2.1 \mathrm{~mm}, 2.6 \mu \mathrm{m})$. Multiplereaction monitoring was selected for detection at $\mathrm{m} / \mathrm{z} 711.4>624.4$. The lower limit of quantification was $1 \mathrm{ng} / \mathrm{ml}$ for mouse studies, and $5 \mathrm{ng} / \mathrm{ml}$ for rat and dog studies. For clinical study, the lower limit of quantification was $10 \mathrm{ng} / \mathrm{ml}$.

\section{Prediction Performances and Parameter Analyses}

Since observed PK data were drug concentrations in plasma, PBPK modelpredicted plasma concentrations were obtained by dividing the predicted blood concentrations by the blood-to-plasma ratio. 
Noncompartmental Analysis. For in vivo studies, the S 55746 plasma concentration-time profiles were analyzed by noncompartmental analysis, and the area under the curve (AUC) was calculated using the log-linear trapezoidal rule.

Assessment of Prediction Accuracy. The accuracy of the PBPK prediction was evaluated on AUC and plasma concentrations. The goodness of prediction in each preclinical species was evaluated based on the average fold error $(A F E)$ and on the root mean square error (RMSE) (Sheiner and Beal, 1981). The fold error, AFE, and RMSE were calculated as follows:

$$
\begin{gathered}
\text { fold error }=\frac{P R E D}{O B S} \\
A F E=10^{\frac{\sum \log \text { fold error }}{n}} \\
R M S E=\sqrt{\frac{\sum(\log P R E D-\log O B S)^{2}}{n}}
\end{gathered}
$$

Where $P R E D$ is the prediction, $O B S$ is the observation, and $n$ is the number of samples.

To account for the interindividual variability existing in clinical data, prediction performances were assessed using the 95th confidence interval of the simulated AUC and $C_{\max }$ (Abduljalil et al., 2014).

\section{Results}

S 55746 Compound. From in vitro experiments (data shown in Table 1), S 55746 was shown to be an ampholyte compound with a high lipophilicity. In all species tested, S 55746 was found to be highly bound to plasma proteins and extensively metabolized (mainly by cytochrome P450 enzymes). Also, S 55746 exhibits both enzymatic induction properties and mechanism-based inhibition on CYP3A4.

PBPK Modeling in Mice. A basic PBPK approach was first applied in mice using the intrinsic clearance parameters determined with in vitro experiments [and extrapolated to in vivo using physiologic scaling factors (SFs)] and distribution parameters predicted from physicochemical properties. This approach resulted in poor prediction accuracy and did not account for the nonlinear elimination process seen in vivo (data not shown).

A comparison with a hybrid PBPK approach (Sayama et al., 2013a,b) was undertaken next. In this case, the in vivo clearance and in vivo volume of distribution determined with a previous compartmental model analysis (Pierrillas et al., 2018) were used to calculate empirical SFs in the PBPK model. Parameters determined from the compartmental analysis are shown in Table 2.

In addition, the method of Poulin and Theil (2002a,b) corrected by Berezhkovskiy (2004) provided better performances for the $K p_{T: p}$ calculation than the Rodgers and Rowland method (Rodgers et al., 2005a,b; Rodgers and Rowland, 2006, 2007) (this method better predicting the volume of distribution for strong bases, whereas $\mathrm{S}$ 55746 is an ampholyte compound).

Regarding elimination, the nonlinear elimination uncovered in the previous compartmental analysis (Pierrillas et al., 2018) was incorporated into the PBPK model: a back calculation of the in vivo clearance parameters was performed to insert these parameters into the PBPK model according to the following relationships (see demonstration in the Appendix):

$$
\begin{gathered}
V_{m}=V_{m, i n t} \\
K_{m}=\frac{K_{m, i n t}}{f_{u, b l}}+\frac{V_{m, i n t}}{Q_{h}}
\end{gathered}
$$

where $V_{m}$ is the in vivo maximum rate of elimination, $V_{m \text {,int }}$ is the intrinsic maximum rate of elimination, $K_{m, i n t}$ is the intrinsic drug concentration corresponding to $50 \%$ of the intrinsic maximum rate of elimination, $\mathrm{f}_{\mathrm{u}, \mathrm{bl}}$ the unbound fraction in blood, and $Q_{h}$ the hepatic blood flow.

In addition, the considered compound was demonstrated to have a low hepatic extraction coefficient $(<0.3)$ during PK studies in mice. Consequently, the in vivo clearance could be approximated by the intrinsic clearance times the unbound fraction:

$$
\begin{aligned}
V_{m} & =V_{m, i n t} \\
K_{m} & =\frac{K_{m, i n t}}{f_{u, b l}} .
\end{aligned}
$$

A predictions versus observations plot of plasma concentrations performed with the hybrid approach is shown in Fig. 2, and all measures of predictive performances are reported in Table 3. PBPK predictions resulting from the hybrid approach and the descriptions of concentration-time profiles (shown in the Supplemental Material) were more satisfactory than those obtained with the classic method (using in vitro $C L_{i n t}$ ). The $\frac{A U C_{\text {Obs }}}{A U C_{\text {Pred }}}$ ratios for each dose are in the range of acceptance ( 0.5 -fold to 2 -fold). In the observed dose range in mice, $A F E$ ranged from 0.672 to 1.42 and $R M S E$ varied from 0.138 to 0.491 . These performances are acceptable because no interindividual variability has been taken into account in the proposed PBPK model. The hybrid approach was therefore selected for use in the interspecies extrapolation, and the subsequent qualification with rat and dog.

Qualification of the PBPK Approach with Rat and Dog Data. As the hybrid approach was satisfactory in mice, SFs for clearance and distribution parameters were calculated from the mouse as follows:

$$
\begin{gathered}
S F_{V_{m}}=\frac{V_{m_{\text {viro }}}}{V_{m}} \\
S F_{K_{m}}=\frac{K_{m_{\text {viro }}}}{K_{m}} \\
S F_{K p}=\frac{V_{s s, v i v o}-V_{b l} \cdot R_{b l: p}}{\sum V_{T} \cdot K p_{T: p}}
\end{gathered}
$$

where $S F_{K m}, S F_{V m}$, and $S F_{K p}$ are respectively the $S F s$ for $K_{m}, V_{m}$, and the partition coefficient $K p ; K_{m}$ vitro and $V_{m \text { vitro }}$ are the clearance parameters determined from in vitro experiment; and $K_{m}, V_{m}$ are the in vivo clearance and distribution parameters determined from the previous compartmental analysis (Pierrillas et al., 2018).

To qualify the hybrid PBPK approach, clearance and distribution parameters were predicted in rat and dog using the SF defined in mice, assuming that the discrepancies between in vitro and in vivo parameters in other species had an origin similar to that in mice. For both species, predictions versus observations plots are presented in Fig. 2, concentrationtime profiles for all of the qualification exercises are presented in Supplemental Fig. 1, and predictive performances are shown in Table 4.

For rat data, concentration-time profiles were well predicted; furthermore, the $\frac{A U C_{O b s}}{A U C_{\text {Pred }}}$ ratios were acceptable $\left(\frac{A U C_{O b s}}{A U C_{\text {Pred }}}\right.$ ratios between

\section{TABLE 2}

In vivo mouse $\mathrm{PK}$ parameters using compartmental $\mathrm{PK}$ approach

\begin{tabular}{lcc}
\hline \multicolumn{1}{c}{ Parameters } & \multicolumn{1}{c}{ Definition } & Estimates \\
\hline $\mathrm{V}_{\mathrm{c}}(\mathrm{l} / \mathrm{kg})$ & $\begin{array}{c}\text { Volume of distribution of the central } \\
\text { compartment } \\
\text { Volume of distribution of the peripheral } \\
\text { compartment }\end{array}$ & 1.18 \\
$\mathrm{~V}_{\mathrm{p}}(1 / \mathrm{kg})$ & $\begin{array}{c}\text { Drug concentration corresponding to } \\
50 \% \text { of maximum rate of elimination }\end{array}$ & 654 \\
$K_{m}(\mathrm{ng} / \mathrm{ml})$ & $\begin{array}{c}\text { Maximum rate of elimination } \\
V_{m}(\mathrm{mg} / \mathrm{h} \text { per kilogram })\end{array}$ & 3.79 \\
\hline
\end{tabular}




\section{Mouse}

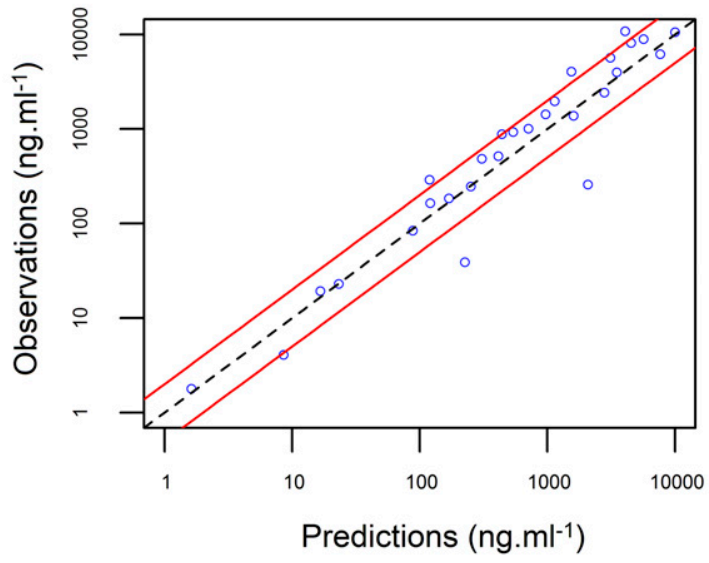

Rat

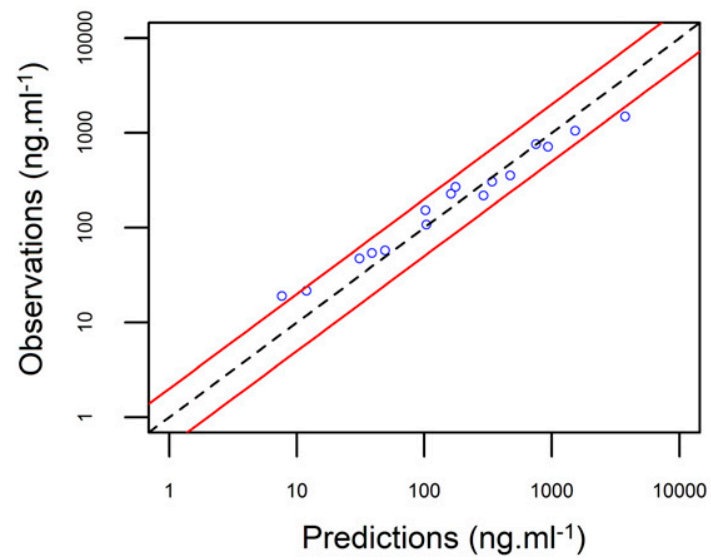

Dog

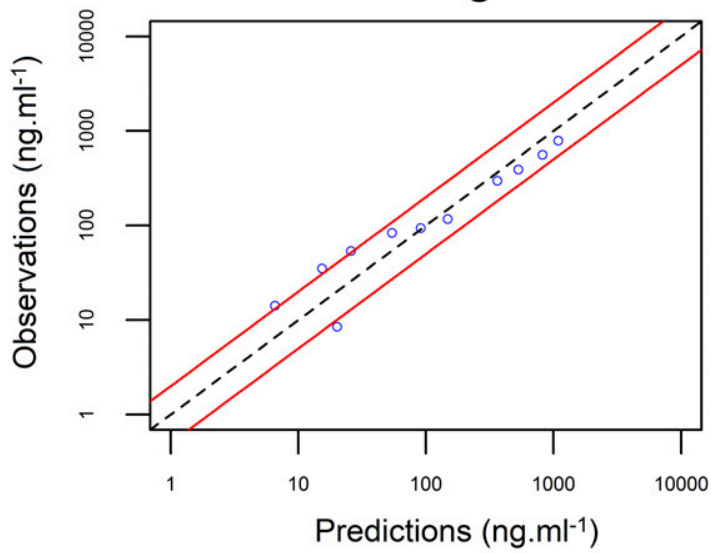

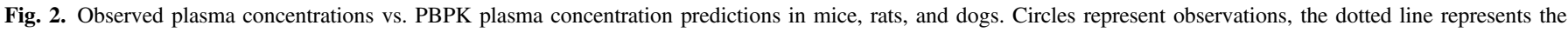
identity line, and the red lines represent the 2-fold interval.

1.10 and 1.20). Concerning dog data, the exposure parameters were also satisfactory $\left(\frac{A U C_{\text {Obs }}}{A U C_{\text {Pred }}}\right.$ ratios less than 2 and above 0.5$)$, but the concentration-time profiles were less well described (especially the terminal phase) than in the rat.

Regarding prediction performances of plasma concentrations, $A F E$ values for both species were not out of the limits of [0.5, 2], and RMSE did not exceed $27.6 \%$. These results implied that the predictions were acceptable.

Thus, the qualification of the hybrid approach with the application of the SFs was considered satisfactory with rat and dog data. For this reason, the same strategy was selected for the prediction of human PKs.

PBPK Predictions Challenged with Clinical Data. The SFs defined in mice were applied to human in vitro clearance and distribution parameters. In addition, the metabolic pathway of S 55746 determined from in vitro studies on human cells was incorporated into the model. In vitro studies highlighted that cytochromes were responsible for $85 \%$ of the metabolism of S 55746 and furthermore that CYP3A4 was the main isoenzyme involved ( $97 \%$ of cytochrome-mediated metabolism) (Table 1). Accordingly, liver clearance was described as the sum of CYP3A4-dependent clearance (82.5\%) and CYP3A4-independent clearance $(17.5 \%)$. Because this CYP3A4-independent clearance likely resulted from uridine $5^{\prime}$-diphospho-glucuronosyltransferase (UGT enzymes), an interindividual variability of $75 \%$ was added to this parameter (Court, 2010). Concerning the absorption process, the dissolution profile of the drug in formulation and the in vitro permeability data from Caco-2 experiments were added to the human PBPK model. The predictions were better using hepatocyte data rather than microsomal data (data not shown), which is certainly due to the involvement of drug conjugation.

For both PK sampling days (day 1 and day 8), the predicted concentration-time profiles were in good agreement with the observed data (Supplemental Fig. 2). Furthermore, despite the large interindividual variability, our approach satisfactorily predicted the observed AUC

TABLE 3

Prediction performances of the PBPK model in mice

\begin{tabular}{|c|c|c|c|}
\hline \multirow{2}{*}{ Dose $(\mathrm{mg} / \mathrm{kg})$} & \multicolumn{3}{|c|}{ AUC } \\
\hline & $\mathrm{AUC}_{\mathrm{Obs}}$ & $\mathrm{AUC}_{\text {Pred }}$ & $\mathrm{AUC}_{\mathrm{Obs} / \text { Pred }}$ Ratio \\
\hline \multicolumn{4}{|c|}{ ng.h/ml } \\
\hline 1 & 437 & 311 & 0.71 \\
\hline 3 & 2020 & 1256 & 0.62 \\
\hline 10 & 9170 & 8032 & 0.88 \\
\hline 25 & 38,846 & 41,616 & 1.07 \\
\hline \multirow{2}{*}{ Dose (mg/kg) } & & \multicolumn{2}{|c|}{ Concentration } \\
\hline & & $A F E$ & RMSE \\
\hline All doses & & 0.865 & 0.303 \\
\hline 1 & & 0.820 & 0.138 \\
\hline 3 & & 0.672 & 0.275 \\
\hline 10 & & 0.911 & 0.324 \\
\hline 25 & & 1.42 & 0.491 \\
\hline
\end{tabular}

Obs, observed; Pred, predicted. 
TABLE 4

Prediction performance of the PBPK model in rat and dog (model qualification)

\begin{tabular}{|c|c|c|c|}
\hline \multirow{2}{*}{ Interspecies qualification performance } & \multicolumn{3}{|c|}{ AUC } \\
\hline & $\mathrm{AUC}_{\mathrm{Obs}}$ & $\mathrm{AUC}_{\text {Pred }}$ & AUC $_{\text {Obs/Pred }}$ Ratio \\
\hline & \multicolumn{2}{|c|}{ ng.h/ml } & \\
\hline \multicolumn{4}{|l|}{ Dose (mg/kg) } \\
\hline \multicolumn{4}{|l|}{ Rat data } \\
\hline 0.5 & 129 & 143 & 1.11 \\
\hline 1.5 & 402 & 462 & 1.15 \\
\hline 5 & 1650 & 1945 & 1.18 \\
\hline \multicolumn{4}{|l|}{ Dog data } \\
\hline 0.2 & 110 & 74 & 0.67 \\
\hline \multirow[t]{3}{*}{1.5} & 908 & 1236 & 1.36 \\
\hline & \multicolumn{3}{|c|}{ Concentration } \\
\hline & $A F E$ & & RMSE \\
\hline \multicolumn{4}{|l|}{ Dose $(\mathrm{mg} / \mathrm{kg})$} \\
\hline \multicolumn{4}{|l|}{ Rat data } \\
\hline All doses & 0.914 & & 0.195 \\
\hline 0.5 & 0.829 & & 0.156 \\
\hline 1.5 & 0.868 & & 0.133 \\
\hline 5 & 1.04 & & 0.256 \\
\hline \multicolumn{4}{|l|}{ Dog data } \\
\hline All doses & 0.955 & & 0.234 \\
\hline 0.2 & 0.57 & & 0.276 \\
\hline 1.5 & 1.467 & & 0.192 \\
\hline
\end{tabular}

and $C_{\max }$ values for patients at all doses (Fig. 3), permitting the qualification of the PBPK extrapolation strategy at eight dose levels. Exploring the PK characteristics of S 55746 in humans by simulation of the PBPK model at the different doses (Supplemental Fig. 3), S 55746 PK appeared to be proportional to the dose in the studied dose range.

\section{Discussion}

A new approach has been developed for predicting the nonlinear PK characteristics in humans of a new Bcl-2 inhibitor using PBPK modeling and interspecies extrapolation. The application of the classic interspecies prediction methods (allometry, Dedrick method, Wajima method) was not appropriate because of the violation of their main assumptions (nonlinear PKs of S 55746 in nonclinical species). Consequently, in vivo mouse data were used to build a hybrid PBPK model, and SFs were derived to correct in vitro clearance $\left(V_{m}, K_{m}\right)$ and distribution $(K p)$ parameters. Since SFs determined in mice were applied to other species, this implied that the reasons for divergence between in vitro and in vivo parameters were similar among species.

The use of in vitro parameters with physiologic SFs directly as input into the PBPK model in mice led to poor prediction accuracy and particularly did not reflect the nonlinear behavior of the compound seen during in vivo preclinical studies along with other factors not taken into account in the commonly used (or physiologic) extrapolation factors. This was accounted for by the development of a hybrid approach defining SFs in the mouse for both $V_{m}$ and $K_{m}$ parameters, and was qualified using two other nonclinical species (rat and dog).

The high value of RMSE found in the model evaluation exercise in mice is probably due to the fact that the Simcyp animal module (version 13) does not incorporate physiologic interindividual variability. In consequence, all of the variability highlighted during the previous compartmental population analysis of in vivo mouse data (Pierrillas et al., 2018) was considered as residual variability in the PBPK analysis and consequently inflated $R M S E$ values.

The use of SFs has always been controversial regarding the rationale and foundations. The need for additional SFs between in vitro and in vivo values for clearance and distribution parameters can arise from many processes. In this example, a likely explanation could be the implication of transporters for the distribution and elimination of the compound. Indeed, during clearance determination on microsome or hepatocyte suspension, transporters are not functional, and consequently, the measurement does not represent the whole of what is occurring in vivo. S 55746 is a BDDCS (Biopharmaceutics Drug Disposition Classification System) class 2 compound (Benet et al., 2011; Broccatelli et al., 2012), so this assumption is reinforced. In addition, approximately $50 \%$ of S 55746 molecules are positively ionized at physiologic $\mathrm{pH}$, confirming the plausibility of the involvement of cation transporters, such as organic cation transporters (Koepsell et al., 2007; Giacomini et al., 2010).

The implication of transporters affecting the distribution process can also be invoked to explain the need for SFs for $K p$ values. Other arguments in favor of SF could be an incorrect assessment of the plasma protein binding (i.e., $\mathrm{f}_{\mathrm{u}, \mathrm{p}}$ ) or nonconsideration of a tissue-binding component in the $K p$ calculation.

Furthermore, the interspecies extrapolation with the hybrid approach was based on the assumption that the reasons for divergence between in vitro and in vivo parameters were similar across species. This can be considered as a strong hypothesis but was supported by the data; however, finding mechanistic explanations for SFs remains a challenge for future research.

Regarding the human data, the best results were obtained using the clearance (in combination with the SFs) determined from hepatocytes rather than microsomes. The possible involvement of conjugation or glucuronidation (which is not assessed with microsome experiments) can explain this result.

In addition, in vitro studies revealed that S 55746 affected its own metabolism (CYP3A4) by mechanism-based inhibition and induction. The predicted combination of both phenomena seems to reflect the observed kinetic profile of S 55746. However, the power to detect an invalidation of the model is low because of the small population size combined with the large observed variability.

On the other hand, for a few patients (over all doses), the PBPK model does not seem to capture $C_{\max }$ and exposure parameters very well. Nevertheless, investigation showed that these patients were also given proton-pump inhibitors; these medicines increase the $\mathrm{pH}$ in the stomach, leading to lower solubility of S 55746 and consequently to a reduction in drug absorption.

Overall, the combination of this PBPK model-building approach with interspecies extrapolation led to acceptable and satisfactory predictions in humans at different doses and described well all of the kinetic processes observed so far. Furthermore, the PBPK model-building strategy could have important applications in the development of drugs with nonlinear kinetics by helping to anticipate PK behavior using all information available from preclinical and in vitro studies at the earliest stage. In addition, the combination of this PBPK model with a pharmacodynamic model could be useful for early forecasting of efficacy in humans, to propose doses for testing in clinical studies, to optimize the design of phase 1 studies, and ultimately for making go/no go decisions.

\section{Appendix: Demonstration of the Relationship between Intrinsic and Whole Body Clearance Parameters}

We know that:

$$
C L_{h, b l}=\frac{Q_{h} \cdot f_{u, b l} \cdot C L_{i n t}}{Q_{h}+f_{u, b l} \cdot C L_{i n t}}
$$

In addition, 


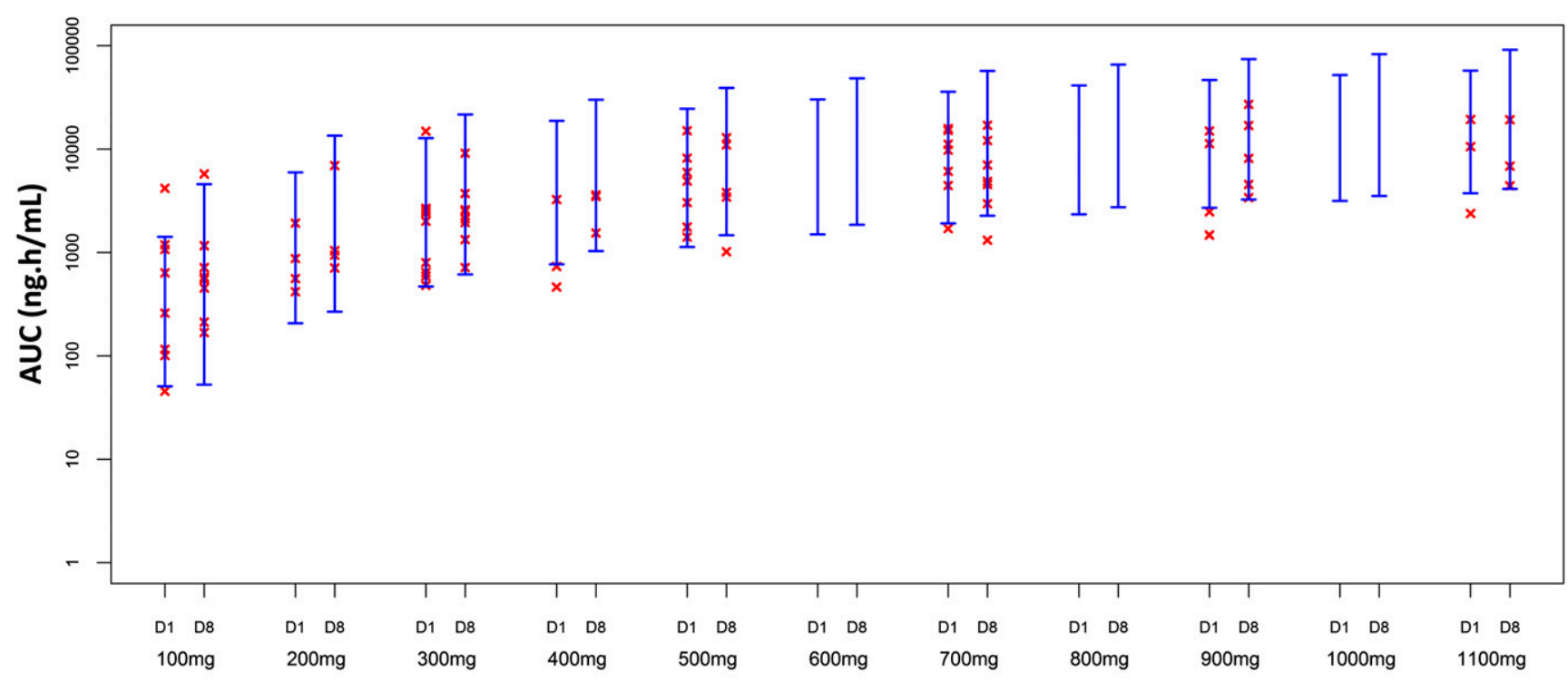

Dose

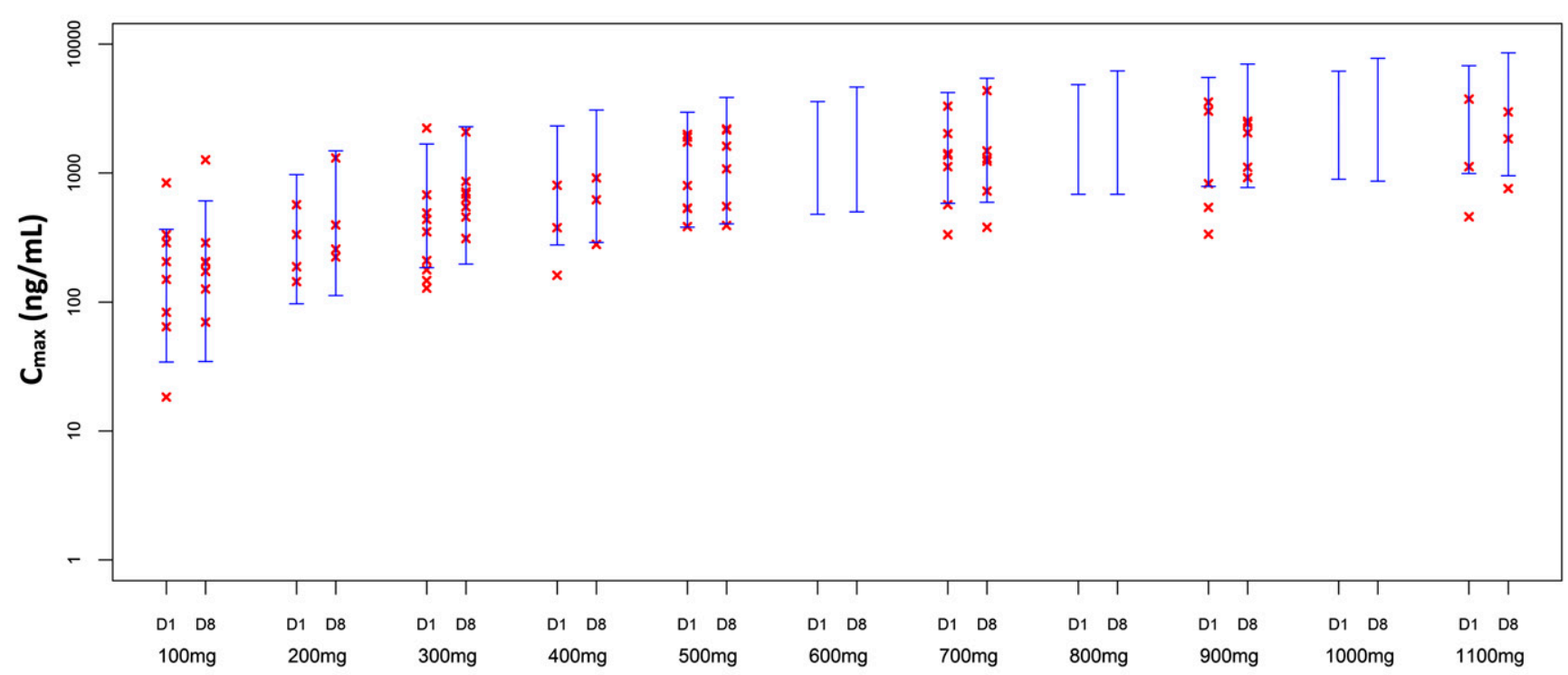

Dose

Fig. 3. AUC and $C_{\max }$ in plasma vs. doses and day of treatment. Crosses represent the observations, and the blue segments represent the $95 \%$ prediction interval of AUC and $C_{\text {max }}$.

$$
C L_{i n t}=\frac{V_{m, i n t}}{K_{m, i n t}+C_{u}}
$$

Therefore, we can write:

$$
\begin{array}{r}
C L_{h, b l}=\frac{Q_{h} \cdot f_{u, b l} \cdot V_{m, i n t}}{K_{m, i n t}+C_{u}} \times \frac{K_{m, i n t}+C_{u}}{Q_{h} \cdot\left(K_{m, i n t}+C_{u}\right)+f_{u, b l} \cdot V_{m, i n t}} \\
C L_{h, b l}=Q_{h} \cdot\left[\frac{f_{u, b l} \cdot V_{m, i n t}}{\left(Q_{h} \cdot K_{m, i n t}+f_{u, b l} \cdot V_{m, i n t}\right)+Q_{h} \cdot C_{u}}\right]
\end{array}
$$

Then,

$$
\begin{gathered}
C L_{h, b l}=\frac{f_{u, b l} \cdot V_{m, i n t}}{\left(K_{m, i n t}+\frac{f_{u, b l} \cdot V_{m, i n t}}{Q_{h}}\right)+C_{b l} \cdot f_{u, b l}} \\
C L_{h, b l}=\frac{V_{m, i n t}}{\left[\frac{K_{m, i n t}}{f_{u, b l}}+\frac{V_{m, i n t}}{Q_{h}}\right]+C_{b l}}
\end{gathered}
$$

Moreover,

$$
C L_{h, b l}=\frac{V_{m}}{K_{m}+C_{b l}}
$$

which permits us to write:

$$
\frac{V_{m}}{K_{m}+C_{b l}}=\frac{V_{m, i n t}}{\left[\frac{K_{m, i n t}}{f_{u, b l}}+\frac{V_{m, i n t}}{Q_{h}}\right]+C_{b l}}
$$

And from this equation we can deduce:

$$
\begin{gathered}
V_{m}=V_{m, i n t} \\
K_{m}=\left[\frac{K_{m, i n t}}{f_{u, b l}}+\frac{V_{m, i n t}}{Q_{h}}\right]
\end{gathered}
$$




\section{Authorship Contributions}

Participated in research design: Pierrillas, Henin, Ogier, Amiel, Kraus-

Berthier, Bouzom, and Tod.

Conducted experiments: Kraus-Berthier.

Performed data analysis: Pierrillas, Henin, and Tod.

Wrote or contributed to the writing of the manuscript: Pierrillas, Henin, Ball, Ogier, Amiel, Chenel, Bouzom, and Tod.

\section{References}

Abduljalil K, Cain T, Humphries H, and Rostami-Hodjegan A (2014) Deciding on success criteria for predictability of pharmacokinetic parameters from in vitro studies: an analysis based on in vivo observations. Drug Metab Dispos 42:1478-1484.

Bagci EZ, Vodovotz Y, Billiar TR, Ermentrout GB, and Bahar I (2006) Bistability in apoptosis: roles of bax, bcl-2, and mitochondrial permeability transition pores. Biophys $J$ 90:1546-1559.

Benet LZ, Broccatelli F, and Oprea TI (2011) BDDCS applied to over 900 drugs. AAPS J 13: 519-547.

Berezhkovskiy LM (2004) Determination of volume of distribution at steady state with complete consideration of the kinetics of protein and tissue binding in linear pharmacokinetics. $J$ Pharm Sci 93:364-374.

Boxenbaum H (1982) Interspecies scaling, allometry, physiological time, and the ground plan of pharmacokinetics. J Pharmacokinet Biopharm 10:201-227.

Broccatelli F, Cruciani G, Benet LZ, and Oprea TI (2012) BDDCS class prediction for new molecular entities. Mol Pharm 9:570-580.

Chen T, Mager DE, and Kagan L (2013) Interspecies modeling and prediction of human exenatide pharmacokinetics. Pharm Res 30:751-760.

Cosulich SC, Worrall V, Hedge PJ, Green S, and Clarke PR (1997) Regulation of apoptosis by BH3 domains in a cell-free system. Curr Biol 7:913-920.

Court MH (2010) Interindividual variability in hepatic drug glucuronidation: studies into the role of age, sex, enzyme inducers, and genetic polymorphism using the human liver bank as a mode system. Drug Metab Rev 42:209-224.

Dedrick RL (1973) Animal scale-up. J Pharmacokinet Biopharm 1:435-461.

Dong JQ, Salinger DH, Endres CJ, Gibbs JP, Hsu CP, Stouch BJ, Hurh E, and Gibbs MA (2011) Quantitative prediction of human pharmacokinetics for monoclonal antibodies: retrospective analysis of monkey as a single species for first-in-human prediction. Clin Pharmacokinet 50: $131-142$.

Espié P, Tytgat D, Sargentini-Maier ML, Poggesi I, and Watelet JB (2009) Physiologically based pharmacokinetics (PBPK). Drug Metab Rev 41:391-407.

Garrett-Mayer E (2006) The continual reassessment method for dose-finding studies: a tutorial. Clin Trials 3:57-71.

Giacomini KM, Huang SM, Tweedie DJ, Benet LZ, Brouwer KL, Chu X, Dahlin A, Evers R, Fischer V, Hillgren KM, et al.; International Transporter Consortium (2010) Membrane transporters in drug development. Nat Rev Drug Discov 9:215-236.

Hilgers AR, Conradi RA, and Burton PS (1990) Caco-2 cell monolayers as a model for drug transport across the intestinal mucosa. Pharm Res 7:902-910.

Houston JB (2013) Prediction of human pharmacokinetics in 2013 and beyond. Drug Metab Dispos 41:1973-1974.

Houston JB and Carlile DJ (1997) Prediction of hepatic clearance from microsomes, hepatocytes, and liver slices. Drug Metab Rev 29:891-922.

Jamei M, Marciniak S, Edwards D, Wragg K, Feng K, Barnett A, and Rostami-Hodjegan A (2013) The simcyp population based simulator: architecture, implementation, and quality assurance. In Silico Pharmacol 1:9.

Jamei M, Marciniak S, Feng K, Barnett A, Tucker G, and Rostami-Hodjegan A (2009a) The Simcyp population-based ADME simulator. Expert Opin Drug Metab Toxicol 5:211-223.

Jamei M, Turner D, Yang J, Neuhoff S, Polak S, Rostami-Hodjegan A, and Tucker G (2009b) Population-based mechanistic prediction of oral drug absorption. AAPS J 11:225-237.

Jones H and Rowland-Yeo K (2013) Basic concepts in physiologically based pharmacokinetic modeling in drug discovery and development. CPT Pharmacometrics Syst Pharmacol 2:e63.

Jones HM, Gardner IB, and Watson KJ (2009) Modelling and PBPK simulation in drug discovery. AAPS J 11:155-166.

Koepsell H, Lips K, and Volk C (2007) Polyspecific organic cation transporters: structure, function, physiological roles, and biopharmaceutical implications. Pharm Res 24:1227-1251.

Kola I and Landis J (2004) Can the pharmaceutical industry reduce attrition rates? Nat Rev Drug Discov 3:711-715.

Michaelis L, Menten ML, Johnson KA, and Goody RS (2011) The original Michaelis constant: translation of the 1913 Michaelis-Menten paper. Biochemistry 50:8264-8269.

Mordenti J (1986) Man versus beast: pharmacokinetic scaling in mammals. J Pharm Sci 75 $1028-1040$.
Nestorov I (2003) Whole body pharmacokinetic models. Clin Pharmacokinet 42:883-908.

Obach RS, Walsky RL, and Venkatakrishnan K (2007) Mechanism-based inactivation of human cytochrome p450 enzymes and the prediction of drug-drug interactions. Drug Metab Dispos 35 246-255.

Ottilie S, Diaz JL, Horne W, Chang J, Wang Y, Wilson G, Chang S, Weeks S, Fritz LC, and Oltersdorf T (1997) Dimerization properties of human BAD. Identification of a BH-3 domain and analysis of its binding to mutant BCL-2 and BCL-XL proteins. J Biol Chem 272 30866-30872.

Pang KS and Rowland M (1977) Hepatic clearance of drugs. I. Theoretical considerations of a "well-stirred" model and a "parallel tube" model. Influence of hepatic blood flow, plasma and blood cell binding, and the hepatocellular enzymatic activity on hepatic drug clearance. $J$ Pharmacokinet Biopharm 5:625-653.

Patel N, Polak S, Jamei M, Rostami-Hodjegan A, and Turner DB (2014) Quantitative prediction of formulation-specific food effects and their population variability from in vitro data with the physiologically-based ADAM model: a case study using the BCS/BDDCS Class II drug nifedipine. Eur J Pharm Sci 57:240-249.

Peters SA (2008) Evaluation of a generic physiologically based pharmacokinetic model for lineshape analysis. Clin Pharmacokinet 47:261-275.

Pierrillas PB, Henin E, Ogier J, Kraus-Berthier L, Chenel M, Bouzom F, and Tod M (2018) Tumor growth inhibition modelling based on receptor occupancy and biomarker activity of a new Bcl-2 inhibitor in mice. J Pharmacol Exp Ther 367:414-424.

Poulin P and Theil FP (2002a) Prediction of pharmacokinetics prior to in vivo studies. 1. Mechanism-based prediction of volume of distribution. J Pharm Sci 91:129-156.

Poulin P and Theil FP (2002b) Prediction of pharmacokinetics prior to in vivo studies. II. Generic physiologically based pharmacokinetic models of drug disposition. J Pharm Sci 91:1358-1370.

Reed JC (2004) Apoptosis mechanisms: implications for cancer drug discovery. Oncology (Williston Park) 18 (13 Suppl 10):11-20.

Rodgers T, Leahy D, and Rowland M (2005a) Physiologically based pharmacokinetic modeling 1 : predicting the tissue distribution of moderate-to-strong bases. J Pharm Sci 94:1259-1276.

Rodgers T, Leahy D, and Rowland M (2005b) Tissue distribution of basic drugs: accounting for enantiomeric, compound and regional differences amongst beta-blocking drugs in rat. J Pharm Sci 94:1237-1248.

Rodgers T and Rowland M (2006) Physiologically based pharmacokinetic modelling 2: predicting the tissue distribution of acids, very weak bases, neutrals and zwitterions. J Pharm Sci 95 : $1238-1257$.

Rodgers T and Rowland M (2007) Mechanistic approaches to volume of distribution predictions: understanding the processes. Pharm Res 24:918-933.

Rostami-Hodjegan A, Tamai I, and Pang KS (2012) Physiologically based pharmacokinetic (PBPK) modeling: it is here to stay! Biopharm Drug Dispos 33:47-50.

Rowland M, Benet LZ, and Graham GG (1973) Clearance concepts in pharmacokinetics. $J$ Pharmacokinet Biopharm 1:123-136.

Sayama H, Komura H, and Kogayu M (2013a) Application of hybrid approach based on empirical and physiological concept for predicting pharmacokinetics in humans--usefulness of exponent on prospective evaluation of predictability. Drug Metab Dispos 41:498-507.

Sayama H, Komura H, Kogayu M, and Iwaki M (2013b) Development of a hybrid physiologically based pharmacokinetic model with drug-specific scaling factors in rat to improve prediction of human pharmacokinetics. J Pharm Sci 102:4193-4204.

Sekiguchi N, Kato M, Takada M, Watanabe H, Takata S, Mitsui T, Aso Y, and Ishigai M (2011) Quantitative prediction of mechanism-based inhibition caused by mibefradil in rats. Drug Metab Dispos 39:1255-1262.

Sheiner LB and Beal SL (1981) Some suggestions for measuring predictive performance. $J$ Pharmacokinet Biopharm 9:503-512.

Sun D, Lennernas H, Welage LS, Barnett JL, Landowski CP, Foster D, Fleisher D, Lee KD, and Amidon GL (2002) Comparison of human duodenum and Caco-2 gene expression profiles for 12,000 gene sequences tags and correlation with permeability of 26 drugs. Pharm Res 19: $1400-1416$.

Tang H and Mayersohn M (2006) A global examination of allometric scaling for predicting human drug clearance and the prediction of large vertical allometry. J Pharm Sci 95:1783-1799.

Wajima T, Yano Y, Fukumura K, and Oguma T (2004) Prediction of human pharmacokinetic profile in animal scale up based on normalizing time course profiles. J Pharm Sci 93:1890-1900.

West GB, Woodruff WH, and Brown JH (2002) Allometric scaling of metabolic rate from molecules and mitochondria to cells and mammals. Proc Natl Acad Sci USA 99 (Suppl 1): 2473-2478.

Address correspondence to: Philippe Pierrillas, EMR 3738, Ciblage Thérapeutique en Oncologie, Faculté de Médecine \& Maïeutique Lyon Sud Charles Mérieux, 165 chemin du Grand Revoyet-BP 12, 69921 Oullins cedex, France. E-mail: philippe.pierrillas@gmail.com 